\title{
STAYING ACTIVE-STAYING SAFE : DEVELOPMENT OF A PHYSICAL ACTIVITY AND FALLS PREVENTION RESOURCE FOR OLDER PEOPLE WHO DWELL IN THE COMMUNITY
}

\author{
Margaret Armstrong, Amanda Bates, Sally Castell and \\ Patricia Krolik \\ Northern Sydney Health Promotion \\ Northern Sydney Area Health Service
}

Staying Active-Staying Safe is an initiative of the Northern Sydney Health Promotion unit and the Safe Communities project in Ryde. The aim of the initiative is to develop a resource that promotes exercises that can be completed at home by the more frail members of the older population. The resource consists of an audiotape and a booklet, which facilitate exercise at a pace and at a level of simplicity that is appropriate for this age group. This article describes the development and evaluation of the resource, and considers the ways in which the resource is useful in increasing physical activity, functional mobility, and self-efficacy in completing everyday tasks.

\section{BACKGROUND}

Health surveys of older people have determined that about one-third of people aged over 65 years, and 50 per cent of those over 80 , have had a fall in the previous 12 months. ${ }^{1,2}$ About 50 per cent of these people will fall again. The injuries sustained-and the subsequent effect on independence, confidence, and quality of life-have a direct effect on the ability of the ageing population to live in the community. ${ }^{3,4}$ Fear of falling and low self-efficacy (that is, the confidence and ability to perform everyday activities) are independent risk factors for functional disability, which correlate with depression and social isolation. ${ }^{3,4}$ These risk factors are identified in approximately 25-49 per cent of older adults, many of whom have not actually fallen. ${ }^{5}$

Research has shown that gentle exercise has value in reducing falls in older people, through increasing lower limb strength, and by improving balance. ${ }^{6}$ Community surveys have determined that up to two-thirds of older adults prefer to exercise as an individual, and studies of home-based programs of physical activity have found the programs to be effective in improving the health of older people. ${ }^{7}$ Home-based programs are particularly pertinent to the more frail community dwellers who are unable to access exercise classes offered in the community. ${ }^{7}$ To prevent falls in older people, the NSW Department of Health recommended in 2001 that plans should be developed at a local level to implement home-based programs in conjunction with community health providers. ${ }^{6}$ By developing a resource of simple, gentle exercise activities, which could be safely completed at home, it is hoped that increased physical activity and safer functional mobility will be achieved.

The Staying Active-Staying Safe audiotape and booklet contain exercises designed to increase strength and mobility of the lower limbs. Side A of the audiotape has seated, gentle exercises and Side B has chair-assisted, standing exercises. Each side of the audiotape takes approximately 20 minutes to complete. The booklet illustrates the exercises in a diagrammatic form, which correspond to the audiotape. The audiotape and booklet both include safety tips and information about risk factors for falls. The exercises were compiled and recorded by a health promotion staff member who is a physiotherapist experienced in the provision of gentle exercise programs for older people.

The partnership that developed the resource was formed between Northern Sydney Health Promotion and the Ryde Safe Communities Falls Working Group, with joint funding provided from the Northern Sydney Area Health Service and Ryde Council. This funding provided for 1,000 tape and booklet packages, which were distributed free of charge to community dwelling residents in the Ryde and Hunters Hill local government areas. The target group for the distribution of the resource was older people who were aged 65 years or over, frail, socially isolated, mostly housebound, unable to access community exercise classes, and who were at risk of falling.

\section{RESOURCE DEVELOPMENT}

Development of the resource was undertaken in a number of phases over approximately two years. The initial phase consisted of trialling draft versions of the tape and booklet with older inpatients in a hospital-based falls prevention group, and with the older community members of the falls working group. Following these trials, the resource was refined, the tape was professionally recorded, and the booklets were printed.

Subsequent phases involved the development of the evaluation tools, including pre- and post-trial questionnaires and modifying assessments tools such as the Tinetti Falls Self Efficacy Scale. ${ }^{8}$ The resource was then piloted in the community over two three-month periods to determine: ease of use, changes in physical activity levels, functional mobility, and any changes in a participant's ability to manage their own self-care. The pilots were co-ordinated by the Northern Sydney Health Promotion unit in conjunction with inpatient allied health staff from Ryde Hospital and occupational therapy students from the University of Sydney. Other partnerships in this process included community allied health staff from Ryde Hospital Aged Care and Rehabilitation Service, Northern Sydney Home Nursing, and Home and Community Care Services. The occupational therapy students were responsible for conducting the assessments and collating the results under the direction of a health promotion supervisor. 


\section{RESULTS}

A total of 29 older people agreed to participate in the community-based pilots. The small sample size was drawn from a larger group of 70 people, who were mostly unable to participate due to lack of access to tape recorders. The collated results from the pilots were averaged and are as follows:

- the majority of participants were female and over 75 years of age;

- 75 per cent were aged between 79-94 years;

- 92 per cent of participants reported finding the tape and booklet instructions easy to follow;

- 89 per cent found the exercises to be the correct degree of difficulty;

- 50 per cent of participants reported increasing their level of physical activity, using the resource 2-7 times per week;

- 64 per cent found the resource useful for staying active in their own home.

The evaluation determined that the participants who used the resource and increased their physical activity also felt more confident in their functional mobility and self-care activities.

\section{DISCUSSION}

The pilot findings support the appropriateness of the resource as a home-based program of gentle exercise. It was indicated that it could be used easily and safely by the target group without intervention by carers or health service providers. Participants reported an increase in their physical activity, and noticed a corresponding increase and improved confidence in their functional mobility and self care. Improvement of confidence and functional mobility are very important in the prevention of falls. A proportion of participants were found to use the tape and booklet together initially, but came to use the booklet alone as they became more familiar with the exercises. As a number of older people may not have access to a tape recorder, this may have implications for how the resource could be used in the future.

When consulting with community service partners during the pilot phases, the resource was found to have a wider potential than just individual home-based use. Responses from the community indicated that aged care day centres, hospitals, retirement villages, and nursing homes, saw the potential for using the resource for group activities and for individual clients at risk of falling. The resource may also have potential for use in rural and remote areas, where access to community-based gentle exercise programs may be more limited than in metropolitan areas. In response to this, Northern Sydney Health Promotion is currently producing the resource commercially, to enable the resource to reach a wider audience.

\section{CONCLUSION}

A fear often expressed by older people, which is supported by the literature, is the potential of falling and sustaining an injury that could reduce their ability to remain independent at home. ${ }^{2,3}$ The use of simple resources such as Staying Active-Staying Safe has the potential to reduce this fear by increasing physical activity and safety through home-based, gentle exercise. The facilitation of actual ability and greater confidence in functional mobility and self-care has important implications for older people in reducing falls and increasing their perceptions of self-efficacy in managing safely in their own homes.

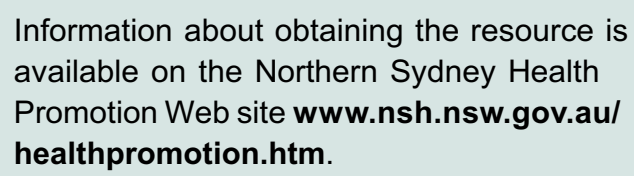

\section{ACKNOWLEDGMENTS}

The authors wish to acknowledge the Ryde Safe Communities Falls Working Group, in-patient and community based allied health staff of Ryde Hospital, occupational therapy students from the University of Sydney, home nurses in Northern Sydney, and community service providers in the Ryde area, for their assistance and support in the development of this resource.

\section{REFERENCES}

1. Kannus P, Parkkari J, Koskinen S, Niemi S, Palvanen M, Jarvinen $\mathrm{M}$ and Vuori I. Fall-Induced Injuries and Deaths Among Older Adults. JAMA 1999; 281(20): 1895-1899.

2. Public Health Division. New South Wales Older People's Health Survey 1999. NSW Public Health Bulletin Supplement. Sydney: NSW Department of Health, 2000.

3. Commonwealth Department of Health and Aged Care. A study into the information needs and perceptions of older Australians concerning falls and their prevention. National Falls Prevention for Older People Initiative 'Step Out with Confidence'. Canberra: Commonwealth Department of Health and Aged Care, 2001.

4. Cheal B, Clemson L. Older People Enhancing their Self Efficacy in Falls Risk Situations. Australian Journal of Occupational Therapy June 2001; 48(2): 80-91.

5. Edelberg, HK. Falls and Function: How to prevent falls and injuries in patients with impaired mobility. Geriatrics 2001; 56(3): 41-48.

6. Public Health Division. Preventing Injuries from Falls in Older People: Background information to assist in the planning and evaluation of local Area-based strategies in NSW. Sydney: NSW Department of Health, 2001.

7. Atienza AA. Home-based physical activity programs for middleaged and older adults: summary of empirical research. Journal of Aging and Physical Activity 2001; 9(Suppl): S38-S58.

8. Tinnetti ME, Williams CS. Falls, Injuries Due to Falls, and the Risk of Admission to a Nursing Home. $N$ Engl J Med 1997; 337(18): 1279-1284. 